\title{
Contemporary Developments in Wireless Sensor Networks
}

\author{
Sangeeta Mittal \\ Department of Computer Science \& Information Technology, Jaypee Institute of Information Technology, Noida, India \\ Email: sangeeta.mittal@jiit.ac.in
}

Alok Aggarwal

Department of Computer Science \& Information Technology, Jaypee Institute of Information Technology, Noida, India Email: alok.aggarwal@jiit.ac.in

\author{
S.L. Maskara \\ G2W - Soura Niloy. 1 - Kailash Ghosh Road.Kolkata - 700 008. ( WB ). India \\ Email: maskara.shankar@gmail.com
}

\begin{abstract}
Wireless Sensor Networks (WSN) since their inception, a decade ago, have grown well in research and implementation. In this work the developments in WSNs are reported in three sub areas of wireless sensor networks that is, wireless sensor node (hardware and software), Communication \& Networking issues in WSNs and application areas. WSNs are characterized by huge data hence research work in aggregation \& mining is also discussed. Contemporary issues of integration of WSNs with other prevalent networks, sensor enabled smartness and role of artificial intelligence methods is elaborated. Insight into future directions \& research avenues in all the above areas is provided.
\end{abstract}

Index Terms -Wireless Sensor Networks, Node, Data Handling, Smart Sensor Networks

\section{INTRODUCTION}

Sensors play very important role in human lives. People want to know about their nearby as well as far away surroundings/environments to take care of any undesirable /dangerous situations and for planning their activities. Man made sensors can be deployed in the places which are required to be explored. A variety of mechanical, thermal, biological, chemical, optical, and magnetic sensors are available for sensing different types of information. The information so sensed has to be converted into digital form and transmitted to appropriate location preferably by wireless means nowadays. In view of the large number of sensors, locations and users it becomes necessary to connect them to form a Wireless Sensor Network (WSN) [1]. A considerable amount of development has been made in WSNs over the last two decades. The advances in wireless sensor networking technologies have been rather very fast, it is therefore felt that the developments in various aspects of the WSN are compiled at one place to get an overall perspective. Three components namely the sensing and processing device called "sensor node", the wireless communication links \& networks and data transmission \& processing form the research areas in this field [2][3]. Each one of them has made a tremendous progress. An analysis of contemporary developments in these sub areas of WSN is the subject matter of this paper and we elaborate on these aspects. The applications of WSN encompass many areas like healthcare, environmental monitoring, smart dwellings and civil \& battle field security. Still challenges such as design of new sensor devices to sense \& calibrate phenomenon of new interests, integration of WSNs with co existing networks and meaningful interpretation of sensor data has to be addressed [4][5].

In this paper a brief description of WSN evolution, the contemporary developments in various aspects of WSN have been discussed to get an up-to-date perspective in the field. For convenience the paper has been discussed in 4 major categories namely related to sensing node, wireless transmission \& networking issues, data handling \& knowledge extraction and emerging applications. The paper is organized as follows. The next section elaborates advances in WSN nodes - hardware \& software and battery technology. Several issues like size, computational power and energy are discussed. In third section the communication and networking issues like ensure coverage and deployment are addressed. Interconnection of WSNs with other networks is also emphasized in this section. Data centricity is one of the features that distinguish WSNs from other wireless networks. The fourth section reviews emerging topics related to efficient transmission and information extraction from WSN data. Fifth section is about a very recent concept of smartness enabled by sensors and use of artificial intelligence in WSNs and in last section emerging applications are enumerated. The paper is then concluded with a discussion on current open challenges to be met.

\section{DEVELOPMENTS IN WIRELESS SENSOR NODE}

The building block of a WSN is the Sensor Node. Wireless Sensor Nodes are fundamental to large scale monitoring of detailed information gathering not possible with any other technique. Sensors are not new to industry 
but have grown in capability with time; the recent sensors are self sufficient devices with components like processor \& memory besides mere sensing and communication. Arrangement of such components in a typical sensor node is shown in figure 1. A typical Sensor node now is characterized by its communication \& sensing range, type of antenna, components, memory, storage, power, computational capability, communication technology, size, programming interface, cost and lifetime. The journey of these wireless sensor nodes is presented here in terms of past, state-of the-art and advancements in hardware and software related to nodes.

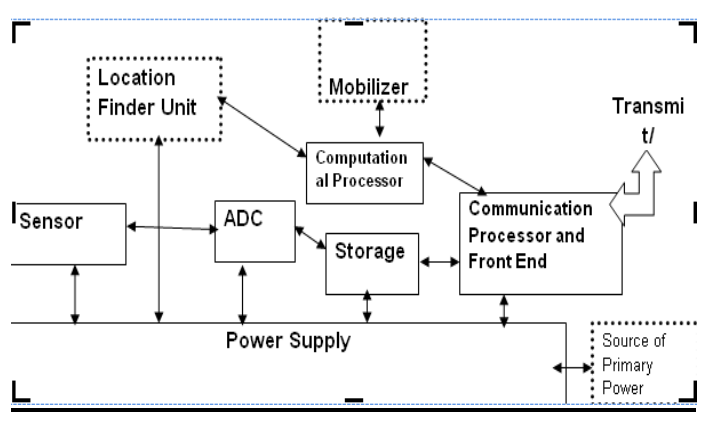

Figure 1: Components of a typical sensor node

In this section we present the developments in sensor node in terms of the advancements in hardware and the amount \& type of software embedded into it.

\section{A. Node Hardware:}

The journey of sensor node hardware in terms of its capabilities and components since their advent till today is summarized as various generations in table 1 . Much before WSN concept, independent non-networked sensors used in automated gates, remote controls and automobiles etc were considered the first generation of sensor hardware. Such sensors were only capable of transmitting analog signals to a controller \&actuator unit. Binary threshold used to decide the action to be taken suffered from lot of false alarms. The problems were addressed by new generation of sensors equipped with capabilities to wirelessly connect with peers. Typical components added to individual sensors were low power
CPU, modest RAM and some secondary storage [6]. Such Wireless Sensors' Network has few tens to thousands of sensor nodes working together to monitor a region to obtain data about the environment. Several families of sensors like MicaZ, smartdust and SunSPOTs are some commercially popular sensing nodes of the second generation.

TABLE I.

GENERATIONS OF WIRELESS SENSOR NODES

\begin{tabular}{|l|l|l|l|}
\hline $\begin{array}{l}\text { Characteristics } \\
\text { of node/network }\end{array}$ & \begin{tabular}{c}
\multicolumn{1}{|c|}{$\mathbf{1}^{\text {st }}$} \\
Generation
\end{tabular} & $\begin{array}{c}2^{\text {nd }} \\
\text { Generation }\end{array}$ & $\begin{array}{l}\mathbf{3}^{\text {rd }} \\
\text { Generation }\end{array}$ \\
\hline $\begin{array}{l}\text { Main Activity of } \\
\text { sensor node }\end{array}$ & $\begin{array}{l}\text { Sensing and } \\
\text { communicat } \\
\text { ion }\end{array}$ & $\begin{array}{l}\text { Previous \& } \\
\text { Numeric } \\
\text { Processing }\end{array}$ & $\begin{array}{l}\text { Previous \& } \\
\text { Logical } \\
\text { Processing }\end{array}$ \\
\hline Topology & Star & Mesh & Hybrid \\
\hline $\begin{array}{l}\text { Processing } \\
\text { capabilities }\end{array}$ & Nil & $\begin{array}{l}\text { Limited to } \\
\text { numeric }\end{array}$ & $\begin{array}{l}\text { Logical - } \\
\text { Abstractions } \\
\text { Inference }\end{array}$ \\
\hline Energy Source & $\begin{array}{l}\text { Constrained } \\
\text { on board } \\
\text { battery }\end{array}$ & Same built & $\begin{array}{l}\text { In Power } \\
\text { harvesters }\end{array}$ \\
\hline Reconfigurable & No & Software & $\begin{array}{l}\text { Hardware \& } \\
\text { Software }\end{array}$ \\
\hline Frequency Band & Infra Red & $\begin{array}{l}433 \quad \text { MHz } \\
\text { and 915 } \\
\text { MHz of } \\
\text { ISM }\end{array}$ & $\begin{array}{l}2.4 \\
\text { (ISM) GHz }\end{array}$ \\
\hline
\end{tabular}

The third generation nodes are futuristic with following capabilities. Micro Power harvesting [7][8]- is scavenging alternative power sources to harvest RF power. Proximate sources of energy like Solar, Temperature Gradients, Human Power, Wind Flow, and Vibrations can be harnessed [9]. Low power circuit designing will further make nodes self sustainable. Reconfigurable Hardware [10] - Use of Field Programmable Gate Arrays (FPGAs) for hardware design reconfiguration and components library to get a new sensor node quickly is being explored. The entire software design will have to be component based to support fast nodes development for various applications.

TABLE II.

COMPONENT SPECIFIC SOFTWARE IN A WSN

\begin{tabular}{|l|l|l|}
\hline \multicolumn{1}{|c|}{ End User } & \multicolumn{1}{|c|}{ Sink } & \multicolumn{1}{c|}{ Nodes/Aggregator Nodes } \\
\hline $\begin{array}{l}\text { Application specific Programming } \\
\text { Abstractions }\end{array}$ & $\begin{array}{l}\text { Programming support for } \\
\text { Simple Object Access Protocol } \\
\text { (SOAP) }\end{array}$ & $\begin{array}{l}\text { Topology control algorithms making } \\
\text { use of routing and aggregation }\end{array}$ \\
\hline Event Assessment software & Query optimization methods & $\begin{array}{l}\text { Application specific I/O interface with } \\
\text { sink }\end{array}$ \\
\hline Data Mining software & Data Intensive Streaming & Sensing control algorithms \\
\hline Security related algorithms/protocols & Pattern recognition algorithms & $\begin{array}{l}\text { Software implementation of Network } \\
\text { stack }\end{array}$ \\
\hline $\begin{array}{l}\text { Software as a Service framework } \\
\text { specific to WSNs }\end{array}$ & $\begin{array}{l}\text { Secure communication } \\
\text { protocols }\end{array}$ & $\begin{array}{l}\text { Secure compression and transmission } \\
\text { algorithms }\end{array}$ \\
\hline Intelligent Decision Support System & $\begin{array}{l}* \text { All above transparent to type } \\
\text { of device }\end{array}$ & $\begin{array}{l}\text { *All above adaptive to uncertain } \\
\text { surroundings }\end{array}$ \\
\hline XML Based Databases & \multicolumn{2}{|l}{} \\
\hline
\end{tabular}


Battery Technology [11][12] - The battery technology has evolved from oldest Nickel - Cadmium heavy batteries to lithium polymer light weight batteries nowadays. The emergence of 'Graphene' based batteries will increase the battery capacity and decrease the weight by huge ratios. Interoperability between sensors from different vendors is an issue due to lack of standardization. This is major hindrance in getting plug $\mathrm{n}$ play nodes in a sensor network.

\section{B. Software in a Sensor Node}

In the current generation of sensors there is a considerable scope of on board processing. It is supported by the software specific to sensor nodes [13]. Software related to WSN can be classified in various categories namely Operating Systems, Programming Languages, Data Management \& Querying Languages, Communication and Security Software. A WSN Operating System is distributed in nodes \& sinks and allows applications to control sensing phenomenon and interrupt routing decisions. WSN operating systems like TinyOS, Contiki, LiteOS and Mantis are representatives in this category [14]. Major OSes for WSNs lack dynamic memory management [15]. Programming languages like NesC are developed as domain specific languages for hardware description, data structures, and data flow representation of WSNs. Incremental Reprogramming protocols that minimize the energy consumption due to reprogramming related transmissions are proposed by [19] and [22]. Currently Deluge protocol is used by TinyOS for reprogramming [21]. WSNs can be viewed as a distributed database and hence at application level database technology for extraction and dissemination of information are required. TinyDB, COUGAR and SINA [16] are few of custom made
DBMS for WSNs. Communication software implementing MAC and networking layer will be discussed in next section. Security software is of paramount importance in view of omnipresence of sensor networks. WSN specific encryption techniques, key Management modules and Intrusion Detection Systems are key areas of security development [17]. Detectionand-classification system developed in VigilNet [18] can detect and classify vehicles, persons, and persons carrying ferrous objects. For quick localization and rendering the data stream usable WSN nodes are implemented as sink, sensing and aggregator nodes[19]. In table 2 software specific to each type of node is listed. Event assessment is done with the help of information stored in XML based databases, using mining tools and decision support systems. The end user is typically connected only to gateway/sink node of WSN implementation of SOAP will render possible the information exchange between sink and remote user possible. The AI related statistical pattern recognition algorithms are deployed for offline queries. In [20], the authors developed a multi level heterogeneous health care solution that provides secured pervasive access to the health care data of the monitored individuals. Some Future Directions for Software Modules in WSN are

- Fusion of local sensor data, predictions and multi rate information from peers in an energy efficient manner

- Development of lightweight encryption mechanisms for new challenges of content rich and real time transmission are required.

- Problem of homomorphism in encryption for encryption in large scale WSNs.

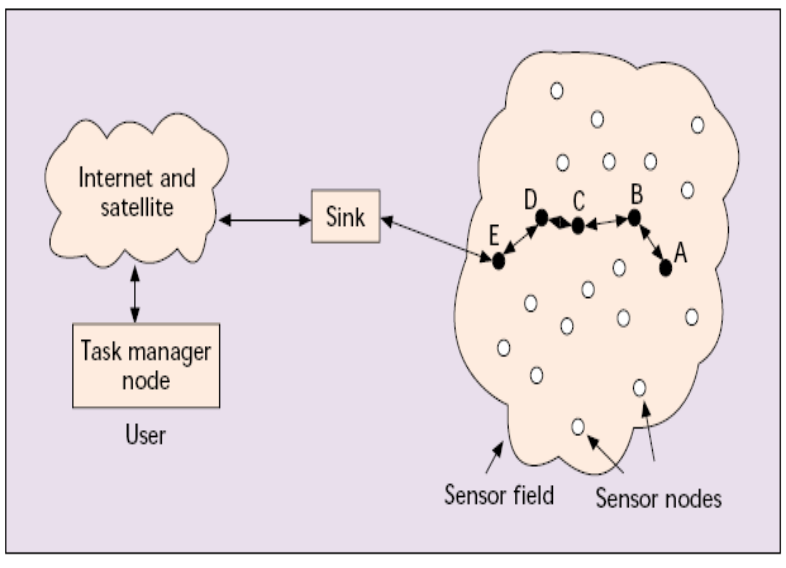

(a)

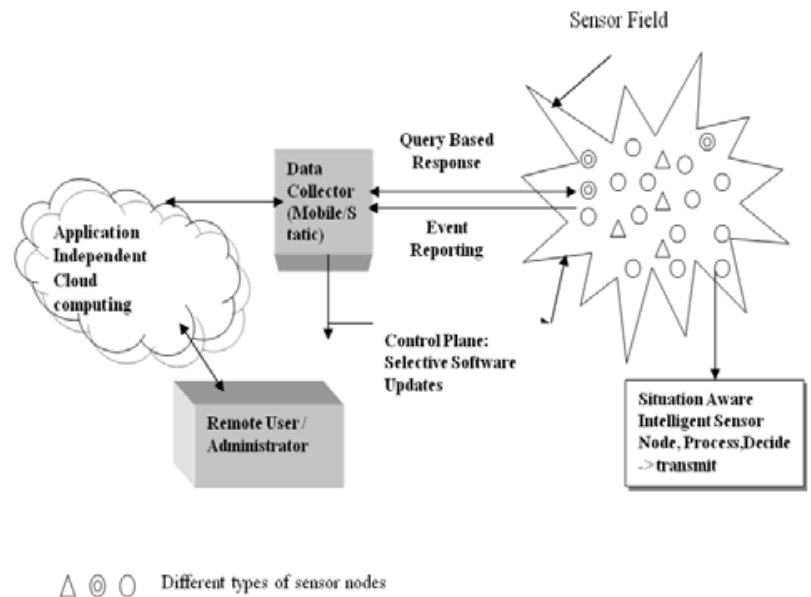

(b)

Figure 2(a) Wireless Sensor Network Communication Architecture [1] b) A Contemporary Architecture [24]

\section{COMMUNICATION AND NETWORKING IN WIRELESS SENSOR NETWORKS}

A key step in exploring the challenges of building a low-power wireless communication platform is to understand the communication mechanisms necessary to construct the multi-hop networks that are envisioned. In 
this section design of communication subsystem is investigated. This is divided into issues related to wireless channel, topology management and actual transmission using medium access and routing methods.. The communication subsystem of the deployed network has to deal with issues related to wireless communication, topology management and routing.

\section{A. Communication}

The wireless medium presents communication issues including interference, noise, path loss, fading and channel correction. Radio components used in a WSN are based on fixed frequencies where sensor nodes within communication range compete for a shared channel to transmit data. Sensor networks need to be supplied with the required amount of bandwidth so that it is able to achieve a minimal required QoS. Limited bandwidth results in congestion which impacts data exchange and leads to packet loss. To communicate over a wireless link, protocols must be built up from the raw electro-magnetic signaling primitives. A transmitter must carefully modulate the RF carrier while receiver performs demodulation and signal analysis. The first step in the communication process is to encode the data for transmission. The coding schemes are designed to increase the probability of a successful transmission by preventing and correcting slight errors. For efficiency reasons the encoding process is pipelined with the actual transmission process. Once the first byte is encoded, transmission may begin. The remaining bytes can be encoded as preceding bytes are transmitted. The physical layer design using MQAM and MPSK modulation schemes are suitable to minimize energy per information bit [23].

\section{B. Networking Issues}

There are some key issues that must be addressed by the network architecture of WSN. In WSNs continual monitoring of channel \& transfer of data to the radio are done in parallel. Secondly, the system must be flexible to meet the wide range of target application scenarios. A typical communication architecture of WSN as shown in fig 2(a) was proposed in [1] and followed by all researchers thereafter. With time, increased functionalities in nodes and application demands; the level and type of communication within the nodes and with the gateway increased. Such a contemporary architecture represented in 2(b), suggested in [24] shows that sensor may transmit either occasionally in query-response mode or continuously in event reporting mode. The sensor node senses the information, processes it to obtain abstractions and then decides whether to transmit, store or discard. The sink acts as gateway and also sends messages to sensor nodes in control plane e.g. software updates and duty cycles. The transmissions to end remote user are done using cloud computing based virtual environments. A suitable set of protocols for realizing this architecture calls for flexible, application specific optimization of communication protocols while obtaining high bandwidth and efficiency. Topology management required to maintain connectivity while conserving energy. Topology awareness due to holes and topology control problems encompassing coverage and connectivity is challenging in WSNs. In mobile networks the problem of topology management is aggravated as compared to static networks. Wang et al. [25] study the deployment schemes for movable sensors. Given an area to be monitored, the proposed distributed self-deployment protocols first discover the existence of coverage holes in the target area then calculate the target positions and move sensors to diminish the coverage holes. Voronoi diagrams [26] are used to discover the coverage holes and three movementassisted sensor deployment protocols VEC, VOR and Minimax are designed.

\section{Link Layer and Routing in WSNs:}

In WSNs, size of sensing area is typically large as compared to short transmission range of sensor nodes. Therefore reliable multi hop wireless communication in presence of energy and computation constraints is main challenge [27]. The actual transmission begins with the execution of a medium access control protocol (MAC) that are are responsible for medium access and error control [28].Various protocols for radio operation control and adaptive duty cycling are 802.11 DCF in PS (Power Saving) Mode and S-MAC [29]. Duty-cycling through topology management by using three layer communication architecture of a large scale WSN and samplers in each layer is described in [30]. Some contemporary MAC protocols proposed to be used with WSNs are 6LowPAN, WiBree, ZigBee, WirelessHART [31]. MAC protocols for new heterogeneous sensor boards need be designed to allow multiple transmitters to share a single communication channel. In a typical WSN application, sensors gather information and transmit the sensed data towards base station through multiple hops [32][33]. In WSNs energy constraints, network dynamics and deployment scale render proactive routing methods infeasible. Early routing methods were Diffusion schemes [11], and opportunistic routing trees to route data to the sink or base-station. Recent research in localization schemes have, however, made it likely that cheap and precise location information can be obtained even in networks where all nodes cannot be GPS enabled [33][34]. Use of information like current total energy of network, priority of data, in taking routing decisions further improve the successful transmissions while decreasing the energy usage [35]. An interesting finding from literature on communication protocols is that they unintentionally categorize WSNs as small scale indoor networks or large/very large scale outdoor networks. The architectural and functional assumptions taken for these two types of networks are summarized as Table 3 above. Most of the assumptions and considerations used in communication and routing related parameters apply to only any one of these. The classification of the application to be developed as being one of these can become the guiding principle for the design of WSN. For example, a routing algorithm that uses extensive message exchanges to find routes is not suitable for small scale networks like body sensor network. On the other hand, 
gateway is generally at one hop distance in small scale WSN but it is not so in large scale ones.

\section{Integration with other networks}

The environments today are populated with lots of wireless networks present concurrently. Varying from application to application a WSN may be surrounded by various wired/wireless networks. The instantaneous connectivity to these networks can elongate the lifetime, connectivity, usability and processing power of the constrained WSNs. Some such interconnection mechanisms are discussed in [36][37] and are also described below.

Integration with IP based Networks will be typically useful when WSN is deployed for event detection [36].The user can be alerted whenever sought event is detected. The user can roam anywhere on an IP based network like LAN or Internet. For instance condition monitoring of equipment in a production plant or delivering patients' health status to doctors. In [38] authors devised an Enterprise Integration Component (EIC) which acts as a mediator between the desktop based application and WSN. The overview of architecture of EIC is given in figure 3.

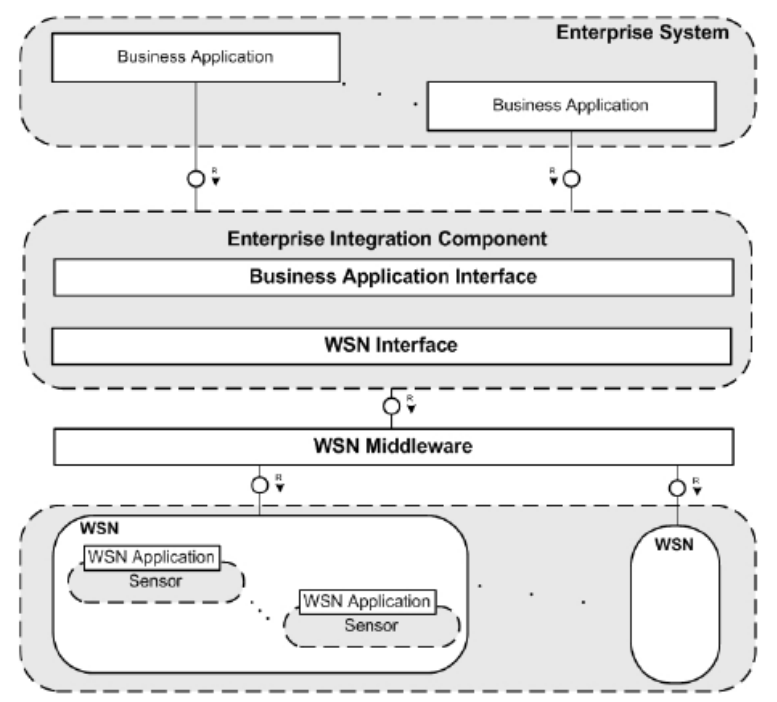

Figure 3: Main Components of Enterprise Integration Component [38]

As in figure 3, the EIC acts as a middleware that allows the business application to directly access the nodes in a secure manner. Use of Web Service standards and ontology for formatting sensor data will further enhance quality and ease of such integration.

Integration with Cellular Networks: is useful in situations where WSN is installed in case of disaster, for long distance communication in such case integration with existing infrastructure of cellular networks will increase the accessibility of WSN data [39].

Integration with Personal Area Networks (PAN): Particularly in Body Sensor Networks WSN data, if routed through a personal device based on PAN technology like Blue tooth which again can be assumed to be available with every person wearing a BSN the distance travelled by sensor data is increased. PAN devices being more resourceful than sensor nodes can transmit the data for further storage or processing .

Seamless Integration: In Autonomous Network Services, plug and unplug of units into any component network is possible without reconfiguration. When a unit breaks or upgrades are required, new units can also be added seamlessly. In [40], for a seamless interconnection with heterogeneous networks an overlay layer is devised that makes the heterogeneity transparent. Ontology based logical architecture will be appropriate for communication in such case. In [24], Ontology enabled semantic web model has been used to propose the implementation of the WSN-as-a-Service concept, the details of sensor nodes is transparent to the end user present at any place in the world. Some Open challenges against achieving this are: In Networking protocols, scalability, fault tolerance, fairness, real time guaranteed delivery, transparency to topology change and reliability issues are still not been addressed fully. MAC layer still has a dearth of strong error detection and correction codes. Heterogeneous sensors on one board may be present hence intra- sensor sleep wake up mechanisms are sought. Transport and application layer protocols are not sufficiently defined for WSNs. In Interconnection protocols for opportunistic bridging of sensor nodes dynamically with any present network still need to be worked upon. Inter - WSN connections and creating backbone of gateway nodes using intermediate IP based network for very wide area networks like whole country border tracking is required.

\section{TECHNIQUES FOR DATA HANDLING}

WSNs are sources of continuous streams of data unless it is used in mere query response mode. The huge volume of data presents challenges in storage, processing, transmission and real time analysis for immediate response [41]. The problems related to data have been handled in literature by various approaches mostly exploiting redundancies and based on any of the following principles: (1) In-network processing for aggregation; as data is highly correlated, not much amount of information is lost [28]. (2) Send only interesting data to the sink, that is, some unusual conditions or event [21]. However the significant event has to be identified by individual nodes or cluster heads. (3) Monitoring Tools that can choose between detailed views of few vs. abstracted view of all dynamically [28]. (4) Data Muling in non adverse environments for relieving nodes from communication. (5) Hybrid approach towards storage where some nodes are nominated for storing a particular type of data and all others with similar type of data only send to that node instead of a common sink [21]. (6) Model-based prediction for adaptive data collection. Authors in [30] show it outperforms event based data collection and in- 
network aggregation in messaging cost, energy consumption and data quality [21]. (7) Structure free approaches of data collection making use of temporal and spatial information of the packets [42]. (8) Implementing adaptive accuracy by dynamically choosing the number of nodes reporting their data and sending others in sleep mode.

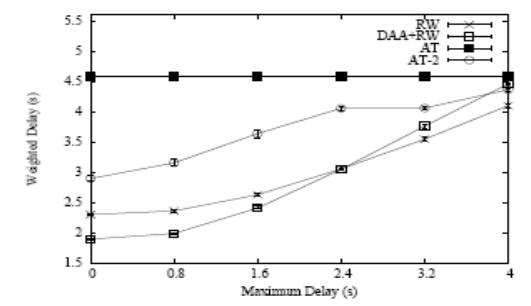

The end-to-end transmission delay v.s. maximum delay.

Figure 4 Improved end to end delay using data aware aggregation using structure free approach [42]

The average delay in structure free approach has shown to be lesser than structured approaches as in figure 3 above. This is because time is not wasted in creating a routing structure and then making the parent nodes wait for the children nodes' data. There are not many empirical results for comparing the above approaches. Knowledge of type of deployment, topology, and purpose, number of nodes and requirement of application whether summaries are enough or detailed data is required can help in deciding which data handling approach to use. Aggregation of data has been based on lossy/lossless and correlation aware functions.

\section{A. WSN Data Stream Mining-Visualizing/Making Use of Data:}

Despite being in large volumes, traditional data mining can't be applied as such in WSNs due to the data being many times redundant, imprecise, missing and continuous valued [43]. The traditional streaming systems like AUROURA don't take into account resource constraints present in sensor systems. This calls for independent methods in sensor stream mining. Fjord is one of the streaming techniques defined specifically for sensor data [43].It makes use of a query processor that processes the query and instructs module controlling relevant sensors to relay their readings. The sensors send aggregated or raw readings to the module. The received data is formulated as tuples and forwarded to the query processor. This scheme, being centralized, doesn't scale well with large number of nodes. Recovering from erroneous data is vital for mining to become basis of a decision support system. Computation intensive heuristic approaches like Bayesian-based estimation to be used for modeling random errors in stream [44][45].

- Formal models of explicit tradeoffs between gains from aggregation and delay \& quality of data.

- DBMS with support of Multi Dimensional spatial - temporal queries over noisy data.

- Analytical modeling of energy consumption in on node processing for aggregation.
- Online mining of multi-rate data stream for real time situation analysis

\section{SMART SEnSING AND SENSING For SMARTNESS}

Recently, the research in WSNs is shifted towards the paradigm of smartness. A smart device doesn't always give same output on a particular input, rather it takes into account the context of execution to modify its output accordingly, just like a human being does. Smartness with respect to WSNs is considered in at least two contexts. First is smartness induced to make WSN operations more efficient and other is WSNs as enablers of creating smart environments i.e. smart sensing and sensing for smartness respectively.

\section{A. Smart Sensing:}

Instead of the node simply reporting every sampled value, an intelligent sensing system can also infer estimations of error and uncertainty, incorporate localized data fusion, and provide event detection. In [18] a multi layer sensing stack as shown in fig 4 above is proposed. The sensing task is here divided into Physical Sensing Layer (PYS) that interfaces with physical sensor hardware by activating sensors and obtaining readings via the node's onboard ADC. Sensor Processing Layer (SPR): processes sensor data obtained from the PYS, in order to provide meaningful sensor reading to higher layers. Sensor Evaluation Layer (SEV): interfaces with the SPR to evaluate the significance of the sensed data and identify faults in the sensor hardware. This includes using event detection techniques and information quantification algorithms to evaluate data, and high level sensor models to identify faults. The SEV provides an interface with the shared application layer, notifying it of detected data and responding to queries regarding the status of the sensors and their most recent values.

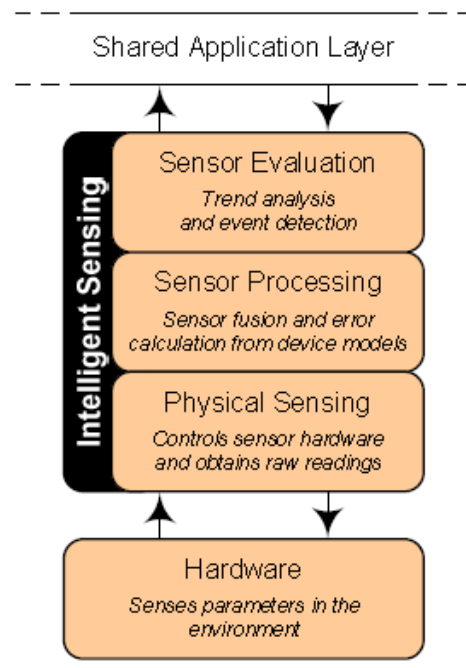

Figure 5 Stack for Multi layer intelligent sensing [46]

Smart sensing also encompasses improving core tasks of WSN by considering current environmental/network conditions [46]. Use of contextual information like remaining energy of network, number of clusters, location of event in basic WSN functions like 
aggregation \& routing can improve the lifetime of the network. Conventionally these decisions were taken statically at the time of network creation or periodically later. With IEEE 1451 based smart sensors being widespread, the whole network can be reorganized using advanced computation intensive methods. A smart sensor has additional functions like signal conditioning \& processing, and decision-making. Inspiration from natural systems like Swarm Intelligence and Quorum Sensing (e.g., colonies of social insects), with aim at optimising distribution of roles and managing network formation and maintenance has been taken [23] to harness the capabilities of smart sensors and improve the performance of WSN. Some preliminary approaches towards this have been in improving routing, synchronization and aggregation [47-52].

\section{B. Sensing for Smartness}

Wireless Sensor Networks act as eyes and ears for context aware systems and are vital to smart environments [35]. Typical processes in a context aware system are Sensing for observing the environment, Fusion - the process of gathering low level data and build abstract and more reliable higher levels of knowledge, Actuation - realisation of response actions and Inference - the ability to build, update and reason about gathered knowledge, inference is important for adaptability, Communication - peer-to-peer, multicast or broadcast communication [23]. Wireless Sensor Network act as an infrastructure for providing contextual information as described in [53]. Using sensing mechanisms like in figure 4, all the processes of Context aware systems as described above can be realized. Mechanisms to assimilate, interpret and make use of context to make the network respond to context are discussed in [53]. It calls for context representation and storage mechanisms for reference and future prediction. The context information gives a compact and more meaningful form of data that can be readily used for event classification and anomaly detection.

\section{C .Artificial Intelligence Techniques: Tools to create Smartness}

Artificial Intelligence (AI) has a significant role to play in delivering smartness for/by WSNs. Many researchers have resorted to AI based tools to provide high quality monitoring \& tracking using WSNs.
TABLE III.

FEW AI TECHNIQUES USED IN WSNS

\begin{tabular}{|c|c|c|}
\hline $\begin{array}{l}\text { Smartness } \\
\text { Aspect }\end{array}$ & $\begin{array}{c}\text { AI Technique } \\
\text { used }\end{array}$ & $\begin{array}{c}\text { Purpose of study \& } \\
\text { Reference }\end{array}$ \\
\hline \multirow{6}{*}{$\begin{array}{l}\text { Smart } \\
\text { Sensing }\end{array}$} & $\begin{array}{c}\text { K-Self } \\
\text { Organizing } \\
\text { Maps } \\
\end{array}$ & $\begin{array}{c}\text { Intelligent Routing } \\
\text { [47] }\end{array}$ \\
\hline & $\begin{array}{c}\text { Swarm } \\
\text { Intelligence }\end{array}$ & $\begin{array}{c}\text { Adaptive Routing } \\
\text { [48] }\end{array}$ \\
\hline & $\begin{array}{c}\text { Fuzzy-ART } \\
\text { neural network }\end{array}$ & $\begin{array}{l}\text { Maximize Network } \\
\text { Lifetime [49] }\end{array}$ \\
\hline & $\begin{array}{c}\text { Self Organizing } \\
\text { Maps (SOMs) }\end{array}$ & $\begin{array}{c}\text { Intelligent } \\
\text { Aggregation [50] }\end{array}$ \\
\hline & $\begin{array}{c}\text { Fuzzy logic } \\
\text { control }\end{array}$ & QoS Management [51] \\
\hline & SOMs & $\begin{array}{c}\text { Clock Synchronization } \\
\text { in WSNs [52] }\end{array}$ \\
\hline \multirow{7}{*}{$\begin{array}{l}\text { Sensing for } \\
\text { Smartness }\end{array}$} & Ontology & Agriculture Grid [54] \\
\hline & $\begin{array}{c}\text { Pattern } \\
\text { recognition and } \\
\text { learning } \\
\end{array}$ & $\begin{array}{c}\text { Continuous Remote } \\
\text { Health Monitoring } \\
\text { [55] }\end{array}$ \\
\hline & $\begin{array}{l}\text { OWL- a method } \\
\text { for defining } \\
\text { ontology }\end{array}$ & $\begin{array}{c}\text { Intelligent Meeting } \\
\text { Room [56] }\end{array}$ \\
\hline & $\begin{array}{c}\text { Back } \\
\text { Propagation } \\
\text { Methods } \\
\end{array}$ & $\begin{array}{l}\text { Intelligent Vehicular } \\
\text { Networks for } \\
\text { Telematic Apps [57] }\end{array}$ \\
\hline & $\begin{array}{l}\text { Temporal } \\
\text { Patterns }\end{array}$ & $\begin{array}{l}\text { Ambient Intelligence } \\
{[58]}\end{array}$ \\
\hline & $\begin{array}{l}\text { Multi Agent } \\
\text { Systems }\end{array}$ & $\begin{array}{c}\text { Commercial Lighting } \\
\text { Control [59] }\end{array}$ \\
\hline & $\begin{array}{l}\text { Hidden Markov } \\
\text { Models }\end{array}$ & $\begin{array}{l}\text { Real Time Daily } \\
\text { Activity Classification } \\
{[60]}\end{array}$ \\
\hline
\end{tabular}

Earlier due to very modest hardware, these computationally intensive methods were not used much in WSNs, but given the advances in related hardware, many researchers have proposed implementation of AI algorithms in sensor nodes. Few examples of use of AI related methods and their purpose are summarized in table 3. In all applications above, the underlying networks are WSNs of varying scale. Besides this AI methods have been used for outlier detection that can be used for recovering from error by predicting values and defying framing attacks by odd one out detection [45]. Concept of smartness in Wireless Sensor Networks is still to be explored for delegation of task to a neighbouring entity if found more capable of handling it. Defining matching and comprehension of situations is a challenging task.

\section{EMERging Application Areas}

WSNs are finding new applications in diversified fields hence posing diversified requirements from the underlying network [62]. Research work has demonstrated great potential for many applications in military target tracking and surveillance [1], natural 
disaster relief [63][64], biomedical health monitoring, and hazardous environment exploration and seismic sensing [65]. Some of these applications are presented in table 6. For example while human centric applications [66-69] demand for higher data rates, military applications [70-72] require advanced tracking capabilities like object classification, an armed vs. an unarmed person. The requirements of WSN are also specific to the application. for example

- Content Rich data transmission in Multimedia Sensor Networks[73-74]

- Adaptation of performance wrt. continuous transmission vs on-demand in Body Sensor Networks [68-69]

- High Mobility of nodes or of data sink in Vehicular Sensor Networks [75-76]

- Real Time Monitoring and highly secure transmission in Military Applications and Health care [77].

Other applications are in various areas like Defense, environment monitoring [78], industrial[79] and human centric, besides this Ambient Intelligence is another new area of WSN applications[80]. Examples include Smart Buildings, Meeting Halls and Home Area Sensor Networks.

\section{A. Open Problems}

One of the major differences between WSNs and other adhoc networks are that every instance of a WSN is highly application dependant. E.g. requirements, challenges and sensing devices for a border perimeter tracking application are entirely different from an underwater WSN [72] and vehicular WSN. Despite lot of research, only recently few networks like monitoring seismic activities [78] and tracking fire equipment [79] are being deployed on actual sites. The real deployment of application will become widespread, if programming abstractions hide the application specific details from user/deplorer of WSN.

\section{Challenges AHEAd}

Design of a suitable WSN is a challenge because many influencing factors such as fault tolerance, scalability, production cost, operating environment, network topology, hardware constraints, transmission media, power consumption and others have to be considered. Right from the breakthrough paper by [1], a number of challenges in practical implementation has been iterated. But unfortunately, in decade long lifetime of WSNs, there has been an inclination towards solving or optimizing only a subset of challenges. Hence some issues foreseen earlier still need to be solved. Moreover due to lot of hype over this technology (many consider it to be surpassing the effect Internet had) for past many years, the expectations in terms of performance and functionality have just heightened. To meet these, provably better algorithms, architectures and systems are required. In view of this, the WSNs of coming years may have to withstand various challenges. For better understanding these has been divided as functional and non functional challenges. The performance of the network is then measured based on quantifiable parameters called performance metrics. This however varies according to need and the nature of WSN.

\section{A. Functional Challenges:}

- Implosion - Remove redundancy in not only similar looking data but semantically similar data also.

- Security \& Privacy -- Prevention of Denial of Service, Key Management and Secure Routing

- Content Rich Sensing - Visual Sensor networks are promising exciting applications in security and health control, it will be required to support high data rate. Meeting Real Time - For the above data to affect further decision process transmission should not be delayed.

- Situation or Context Aware Aggregation/ Routing - New applications are built around situation awareness and predictability to provide user an ambient experience.

- Aggregating Heterogeneous Data- Use of different type of sensors for multi dimensional sensing is required. Aggregation needs to address this issue

- Mobility - for example in vehicular sensor networks for intelligent transportation enhances usability.

- Accuracy - Accurate sensing, processing and transmission is a multi layer challenge.

\section{B. Non Functional Challenges}

These challenges are about execution qualities, such as security, usability, testability, maintainability, extensibility and scalability of WSN. Some challenges are

- Multi Tasking with Resource Management - Being a physically constrained device, a sensor node should individually and collaboratively be able to efficiently manage its resources like energy, processing power and memory to provide maximum throughput and maximize life time.

Space Complexity - To meet hard real time requirements, analysis of algorithm for providing bounds on memory requirements is required. As like other resources memory is also an important and scarce resource.

Worst Case Energy Consumption - Energy consumption in WSNs is a key design constraint. Before implementing a protocol, its energy analysis should be done so that network life time can be predicted and tasks are planned to get an optimal schedule worked out.

Adaptive and Reliable Services- Mean Time Between Failures Till now, adaptive nature of WSNs has been discussed keeping in mind the energy constraint. But with the next generation applications, adaptive would be 
required for reliable delivery of data in wake of time varying demands. Problem of classifying traffic according to its QoS requirements should also be enquired.

Resilience is the ability to provide and maintain an acceptable level of service in the face of faults and challenges to normal operation

\section{Performance Challenges:}

System Lifetime: System lifetime is exhibits by number of rounds in simulation which determines the longevity of the nodes. Lifetime is the most crucial thing in SN so from all aspects of the node and the network, energy has to be utilized in the most effective way possible.

Latency: Data resulted from triggered events are generally time sensitive, however in most of the cases periodic sensing is okay to implement for resulting latency comes in a fraction of a second.

Quality: End user never requires all the data, the only data about the events are processed in BS for analysis. So quality of the network is determined by the quality of data aggregation scheme and protocols implements besides the specific need of the application used.

Delay and Delay Variation: Delay measures time to send and receive a unicast packet from one node to another while the variation of a delay metric with respect to some reference value is determined with delay variation. In sensor networks, delay in data collection from nodes to the BS and interpretation in the BS are concerned with delay.

Bandwidth, Capacity and Throughput: These indicate the capacity of data which can be sent over a link within a given time, however since the data size is very small bandwidth rarely matters.

Hop Count: No of hop in communication determine the cost of path, and eventually the energy consumed in the process.

Signal Strength: SNR as an indication for the link quality and the distance between two nodes is helpful to compute and determine the nodes and their reachability during the communication process.

Ease of Deployment: Sensor network consist of few to several hundred sensor nodes, and these nodes have to be able to communicate with each other even in absence of established network infrastructure.

These issues need to be addressed in a provable manner. More ambitious would be to provide a generic design framework to the application developer such that any application can be built easily and efficiently on top of it by merely assigning his desired performance parameters' values.

\section{CONCLUSION}

Wireless sensor networks are envisioned to become the machinery on top of which ubiquitous/pervasive computing operates. In this paper, we reported how Wireless Sensor Networks have emerged out of their inherent constraints in terms of more powerful nodes.
The advances in communication technology, data handling approaches and software have been discussed. The paradigm of design of WSNs now needs to be shifted from being Constraint Centric to Use Centric. This shift to be realized in terms of smart WSNs was described. It is concluded that two contexts of research demand current attention: (i) design and development of new networking techniques suitable for rapid deployment in ad hoc manner in dynamic environments as well as plug $n$ play in known environments (ii) Extraction of meaningful, reliable and timely information from the deployed network. These two become more complex to achieve when mobility of nodes or sink is also to be taken into account.

\section{References}

[1] Conner, Margery, "Advances in energy-storage technology power wireless devices,” EDN, Feb 3, 2011, pg 26.

[2] Roundy, Shad et al, "Power Sources for Wireless Sensor Networks”, Wireless Sensor Networks, 2004, Pg 1-17.

[3] Chen, G., Hanson, S., Blaauw, D., Sylvester, D., "Circuit Design Advances for Wireless Sensing Applications", Proceedings of the IEEE, On page(s): 1808 - 1827, Volume: 98 Issue: 11, Nov. 2010

[4] Sudevalayam, S.; Kulkarni, P, "Energy Harvesting Sensor Nodes: Survey and Implications," Communications Surveys \& Tutorials, IEEE , vol.PP, no.99, pp.1-19.

[5] Greene, D. Harrish, D. Kalp, and W. Tauche, Making Wireless Sensor Networks Truly Wireless Using RF Power, http://sensormgmt.com/Articles/Powered By\%20FireFly60614.pdf

[6] A. E. Kateeb and L. Azzawi, "Hardware Reconfiguration Capability for Third Generation Sensor Nodes: Design and Challenges”, In Proceedings of the 22nd international Conference on Advanced information Networking and Applications - Workshops, Washington, DC, 675-680, 2008.

[7] Felipe M. Costa, "Energy-Efficient Physical Layer Design for Wireless Sensor Network Links”, In Proceedings of the ICC 2011.

[8] I.F. Akyildiz, W. Su, Y. Sankarasubramaniam, and E. Cayirci, “A Survey on Sensor Networks," IEEE Communication Magazine, August 2002.

[9] Buratti, C.; Conti, A.; Dardari, D.; Verdone, R. An Overview on Wireless Sensor Networks Technology and Evolution. Sensors 2009, 9, 6869-6896.

[10] Xia,F. Wireless Sensor Technology and Applications, Sensors 2009,9, 8824-8830.

[11] Neelam Srivastava, "Challenges of Next-Generation Wireless Sensor Networks and its impact on Society", Journal of Telecommunications, Volume 1, Issue 1, pp128-133, February 2010.

[12] Estrin, D.; Girod, L.; Pottie, G.; Srivastava, M., "Instrumenting the world with wireless sensor 
networks," Acoustics, Speech, and Signal Processing, 2001. Proceedings. (ICASSP '01). 2001 IEEE International Conference on , vol.4, no., pp.20332036 vol.4, 2001.

[13] Ringwald, M.; Romer, K.; , "Deployment of Sensor Networks: Problems and Passive Inspection," Intelligent Solutions in Embedded Systems, 2007 Fifth Workshop on , vol., no., pp.179-192, 21-22 June 2007

[14] Dietrich, I. and Dressler, F. 2009. On the lifetime of wireless sensor networks. ACM Trans. Sen. Netw. 5, 1, Article 5 (February 2009), 39 pages.

[15] C Chong, S.P. Kumar, "Sensor networks: evolution, opportunities, and challenges," Proceedings of the IEEE , vol.91, no.8, pp. 1247- 1256, Aug. 2003.

[16] Romer K, “Tracking Real World phenomenon with smart dust”, proceedings of EWSN'04, pg 28-43, LNCS Springer Verlag.

[17] Shih-Lun Chen; Ho-Yin Lee; Chiung-An Chen; Hong-Yi Huang; Ching-Hsing Luo; , "Wireless Body Sensor Network With Adaptive Low-Power Design for Biometrics and Healthcare Applications," Systems Journal, IEEE , vol.3, no.4, pp.398-409, Dec. 2009.

[18] Obidul Islam and Sajid Hussain. 2006. An Intelligent Multi-hop Routing for Wireless Sensor Networks. In Proceedings of the 2006 IEEE/WIC/ACM international conference on Web Intelligence and Intelligent Agent Technology (WI-IATW '06). IEEE Computer Society, Washington, DC, USA, 239-242.

[19] Kai-Wei Fan, Sha Liu, and Prasun Sinha. 2007. Structure-Free Data Aggregation in Sensor Networks. IEEE Transactions on Mobile Computing 6, 8 (August 2007), 929-942.

[20] D. Ganesan, A. Cerpa, W. Ye, Y. Yu, J. Zhao, and D. Estrin, "Networking Issues in Wireless Sensor Networks", Journal of Parallel and Distributed Computing (JPDC), PP 799-814, Vol 64,July 2007.

[21] B. Gedik, L. Liu, P. S. Yu, "ASAP: An Adaptive Sampling Approach to Data Collection in Sensor Networks", IEEE Trans. Parallel Distributed Systems, 18 (12), Dec. 2007.

[22] Huang, Y.M.; Hsieh, M.Y.; Chao, H.C.; Hung, S.H.; Park, J.H.; , "Pervasive, secure access to a hierarchical sensor-based healthcare monitoring architecture in wireless heterogeneous networks," Selected Areas in Communications, IEEE Journal on , vol.27, no.4, pp.400-411, May 2009.

[23] Xianjin Zhu; Gupta, H.; Bin Tang; , "Join of Multiple Data Streams in Sensor Networks," Knowledge and Data Engineering, IEEE Transactions on , vol.21, no.12, pp.1722-1736, Dec. 2009

[24] Zhang, Y; Meratnia, N; Havinga, P; , "Outlier Detection Techniques for Wireless Sensor Networks: A Survey," Communications Surveys \& Tutorials, IEEE , vol.PP, no.99, pp.1-12,2010.

[25] L. Wang, C. Wang and C. Liu, "Optimal Number of Clusters in Dense Wireless Sensor Networks: A Cross- Layer Approach," IEEE Transactions on
Vehicular Technology, Vol. 58, No. 2, 2009, pp. 966-976.

[26] L. Almazaydeh, E. Abdelfattah, M. Al-Bzoor and A. Al- Rahayfeh, "Performance Evaluation Of Routing Protocols in Wireless Sensor Networks," International Journal of Computer Science and Information Technology, Vol. 2, No. 2, 2010, pp. 64-73.

[27] Eduardo F. Nakamura, Antonio A. F. Loureiro, and Alejandro C. Frery. 2007. Information fusion for wireless sensor networks: Methods, models, and classifications. ACM Comput. Surv. 39, 3, Article 9 (September 2007).

[28] V. Rajendran, K. Obraczka, J.J. Garcia-Luna-Aceves, Energy Efficient, collision-free medium access control for wireless sensor networks, in: Proceedings of the First International Conference on Embedded Networked Sensor Systems (Sensys), Los Angeles, CA, 2003.

[29] Huang, G., Zhang, Y., He, J., and Cao, J. "Fault Tolerance in Data Gathering Wireless Sensor Networks." The Computer Journal, vol. 54 / 6, pp. 976-987, 2011.

[30] Mark D. Krasniewski, Rajesh Krishna Panta, Saurabh Bagchi, Chin-Lung Yang, and William J. Chappell. 2008. Energy-efficient on-demand reprogramming of large-scale sensor networks. ACM Trans. Sen. Netw. 4, 1, Article 2 (February 2008), 38 pages.

[31] R. Sugihara and R. K. Gupta, "Programming models for sensor networks: A survey”, ACM Trans. Sen. Netw. 4, 2, 1-29, Mar. 2008.

[32] M. M. Mozumdar, L. Lavagno, and L. Vanzago,“ A comparison of software platforms for wireless sensor networks: MANTIS, TinyOS, and ZigBee”, ACM Trans. Embed. Comput. Syst. 8, 2 (Jan. 2009), 1-23.

[33] Wang MM, Cao JN, Li J et al. Middleware for wireless sensor networks: A survey. Journal of Computer Science And Technology 23(3): 305-326 May 2008

[34] Panta, R. K., Bagchi, S., and Midkiff, S. P. 2011. Efficient incremental code update for sensor networks. ACM Trans. Sensor Netw. 7, 4, Article 30 (February 2011), 32 pages.

[35] JEONG, J. AND CULLER, D. 2004. Incremental network programming for wireless sensors. In Proceedings of the IEEE International Conference on Sensor and Ad Hoc Communications and Networks (SECON'04). 25-33.

[36] Adi Mallikarjuna V. Reddy, A.V.U. Phani Kumar, D. Janakiram, and G. Ashok Kumar. 2009. Wireless sensor network operating systems; a survey. Int. J. Sen. Netw. 5, 4 (August 2009), 236-255.

[37] M. O. Farooq and T. Kunz, "Operating systems for wireless sensor networks: A survey," Sensors, vol. 11, no. 6, pp. 5900-5930, May 2011. [Online]

[38] Xiangqian Chen; Makki, K.; Kang Yen; Pissinou, N.; , "Sensor network security: a survey," Communications Surveys \& Tutorials, IEEE , vol.11, no.2, pp.52-73, Second Quarter 2009. 
[39] E. Fasolo, M. Rossi, J. Widmer, and M. Zorzi. Innetwork aggregation techniques for wireless sensor networks: a survey. IEEE Wireless Communications, 2(2):70-87, April 2007.

[40] G. F. Carlos, H. Pablo, G. Joaquín and Jesús A. Pérez-Díaz, "Wireless Sensor Networks and Applications: a Survey”, IJCSNS International Journal of Computer Science and Network Security, VOL.7 No.3, March 2007.

[41] S. Diamond and M. Ceruti, “Application of Wireless Sensor Network to Military Information Integration," Proceedings of the 5th IEEE International Conference on Industrial Informatics, Vol. 1, 2007, pp. 317-322.

[42] Freitas, E.P.; Allgayer, R.S.; Wehrmeister, M.A.; Pereira, C.E.; Larsson, T.; , "Supporting platform for heterogeneous sensor network operation based on unmanned vehicles systems and wireless sensor nodes," Intelligent Vehicles Symposium, 2009 IEEE , vol., no., pp.786-791, 3-5 June 2009.

[43] Kong J, Cui J., Wu D., Gerla M., (2005), “Building underwater Ad Hoc networks and sensor networks for large scale real-time aquatic applications”, Military Communications Conference (MILCOM'05), Atlantic City, New Jersey,USA.

[44] Saha, S.; Matsumoto, M.; , "A Wireless Sensor Network Protocol for Disaster Management," Information, Decision and Control, 2007. IDC '07 , vol., no., pp.209-213, 12-14 Feb. 2007

[45] J. Zhou, D. De Roure, "FloodNet: Coupling Adaptive Sampling with Energy Aware Routing in a Flood Warning System”, Journal of Computer Science and Technology, Vol. 22, N. 1, pp. 121-130, January 2007.

[46] H. Mohamed and B. Majid, "Forest Fire Modeling and Early Detection using Wireless Sensor Network" in Ad Hoc \& Sensor Wireless Networks, Vol 7, Philadelphia: Old City Publishing, 2009, pp. 169224.

[47] X. Shen, Z. Wang, and Y. Sun, "Wireless Sensor Networks for Industrial Applications," 5th World Congress on Intelligent Control and Automation, Vol. 4, 2004.

[48] Vivek Katiyar, Prashant Kumar and Narottam Chand. Article: An Intelligent Transportation Systems Architecture using Wireless Sensor Networks. International Journal of Computer Applications 14(2):22-26, January 2011.

[49] Mounib Khanafer, Mouhcine Guennoun, and Hussein T. Mouftah. 2009. WSN architectures for intelligent transportation systems. In Proceedings of the 3rd international conference on New technologies, mobility and security (NTMS'09), Khaldoun AI-Agba, Mohamad Badra, and Gregory B. Newby (Eds.). IEEE Press, Piscataway, NJ, USA, 425-432.

[50] M. McGrath and T. Dishongh, "Wireless Sensor Networks for Healthcare Applications,” Artech House, London, 2010.
[51] Zatout, Y., Campo, E., and Llibre, J. 2009. Toward hybrid WSN architectures for monitoring people at home. In Proceedings of the international Conference on Management of Emergent Digital Ecosystems (France, October 27 - 30, 2009). MEDES '09. ACM, New York, NY, 308-314.

[52] Shih-Lun Chen; Ho-Yin Lee; Chiung-An Chen; Hong-Yi Huang; Ching-Hsing Luo; , "Wireless Body Sensor Network With Adaptive Low-Power Design for Biometrics and Healthcare Applications," Systems Journal, IEEE , vol.3, no.4, pp.398-409, Dec. 2009

[53] S. B. Eisenman, E. Miluzzo, N. D. Lane, R. A. Peterson, G-S. Ahn, and A. T. Campbell. 2007. The BikeNet mobile sensing system for cyclist experience mapping. In Proceedings of the 5th international conference on Embedded networked sensor systems (SenSys '07). ACM, New York, NY, USA, 87-101.

[54] A. Wood, J. Stankovic, G. Virone, L. Selavo, Z. He, Q. Cao, T. Doan, Y. Wu, L. Fang, R. Stoleru, "Context-Aware Wireless Sensor Networks for Assisted-Living and Residential Monitoring," IEEE Network, 22(4):26-33, July-August 2008.

[55] Hoon Kim, Young-Jun Jeon, and Seung-Ho Shin. 2008. Platform of Rich Internet Application for Wireless Sensor Network. In Proceedings of the 2008 International Conference on Computer Science and Software Engineering - Volume 02 (CSSE '08), Vol. 2. IEEE Computer Society, Washington, DC, USA, 531-536.

[56] Paolo Pagano, Francesco Piga, and Yao Liang. 2009. Real-time multi-view vision systems using WSNs. In Proceedings of the 2009 ACM symposium on Applied Computing (SAC '09). ACM, New York, NY, USA, 2191-2196.

[57] 2011-09-15; http://www.sensorsmag.com/specialtymarkets/environmental-monitoring/news/ussiannounces-sale-seismic-system-8966.

[58] May 17 ,

2011; http://www.sensorsmag.com/networkingcommunications/wireless-sensor/wireless-sensornetworks-fire-extinguisher-monitoring-8572.

[59] Simic, S.N.; , "A learning-theory approach to sensor networks," Pervasive Computing, IEEE , vol.2, no.4, pp. 44- 49, Oct.-Dec. 2003.

[60] K. Shin, A. Abraham, and S. Han, "Self organizing sensor networks using intelligent clustering," in Proc. 6th International Conference on Computational Science and its Applications. Glasgow: Springer, Berlin, 8-11 May 2006, pp. 40-49.

[61] Markus C. Huebscher, Julie A. McCann, and Asher Hoskins. 2007. Context as autonomic intelligence in a ubiquitous computing environment. Int. J. Internet Protoc. Technol. 2, 1 (December 2007), 30-39.

[62] Salkham, A., Cunningham, R., Senart, A., Cahill, V.: A Taxonomy of Collaborative Context-Aware Systems. In: Proceedings of the Workshop on Ubiquitous Mobile Information and Collaboration Systems (UMICS), pp. 899-911, at CAISE'06, 
Luxembourg, Grand-Duchy of Luxembourg, 2006, jun, TCD-CS-2006-30

[63] Honggang Wang; Dongming Peng; Wei Wang; Sharif, H.; Hsiao-hwa Chen; Khoynezhad, A.; , "Resource-aware secure ECG healthcare monitoring through body sensor networks," Wireless Communications, IEEE , vol.17, no.1, pp.12-19, February 2010.

[64] GuangZhong Yang, "Cognitive sensing for body sensor networks" Sensors, 2008 IEEE (2008) pp 2629.

[65] Hooman Tahayori, E. Pagani, G. Degli Antoni, S. Astaneh, "Enhanced Sensor Network: A Specialized Infrastructure For Context-Aware Applications”, IADIS Applied Computing, pp. 568-572, Spain, 2007.

[66] Shih-Lun Chen; Ho-Yin Lee; Chiung-An Chen; Hong-Yi Huang; Ching-Hsing Luo; , "Wireless Body Sensor Network With Adaptive Low-Power Design for Biometrics and Healthcare Applications," Systems Journal, IEEE , vol.3, no.4, pp.398-409, Dec. 2009.

[67] Yoosoo Oh; Jonghyun Han; Woontack Woo; , "A context management architecture for large-scale smart environments," Communications Magazine, IEEE , vol.48, no.3, pp.118-126, March 2010

[68] A Wireless Sensor Network Protocol for Disaster Management

[69] Julio Barbancho, Carlos Le, F. J. Molina, and Antonio Barbancho. Using artificial intelligence in routing schemes for wireless networks. Comput. Commun. 30, 14-15 (October 2007), 2802-2811. 2007.

[70] Wang C, Lin Q (2008). Swarm Intelligence Optimization Based Routing Algorithm for Wireless Sensor Networks, International Conference on Neural Networks and Signal Processing, , Nanjing, China June 8 10, pp. 136-141

[71] Mario Cordina and Carl J. Debono. 2009. Maximizing the lifetime of wireless sensor networks through intelligent clustering and data reduction techniques. In Proceedings of the 2009 IEEE conference on Wireless Communications I\& Networking Conference (WCNC'09).

[72] S. Lee and T. Chung. Data aggregation for wireless sensor networks using self-organizing map. AIS 2004, pages 508-517 2005.

[73] Xia, F.; Zhao, W.; Sun, Y.; Tian, Y.-C. Fuzzy Logic Control Based QoS Management in Wireless Sensor/Actuator Networks.Sensors 2007, 7, 31793191.

[74] PALADINA, L., BIUNDO, A., SCARPA, M., PULIAFITO, A.. Artificial Intelligence and Synchronization in Wireless Sensor Networks. Journal of Networks, North America, 4, aug. 2009.

[75] A. Rehman, A. Zubair", Towards Design of ContextAware Sensor Grid Framework for Agriculture", Proceedings of world academy of science, engineering and technology volume 28 april 2008
[76] HARMONI: Context-aware Filtering of Sensor Data for Continuous Remote Health Monitoring Mohomed, Misra, et al. - 2009

[77] H. Chen, T. Finin, and A. Joshi. Semantic web in a pervasive Context-Aware architecture, 2003.

[78] Ansar-Ul-Haque Yasar, Davy Preuveneers and Yolande Berbers (2010). Modelling and Simulating Large Scale Vehicular Networks for Smart Contextaware Telematic Applications, Modelling Simulation and Optimization, Gregorio Romero Rey and Luisa Martinez Muneta (Ed.), ISBN: 978-953-307-048-3, InTech,

[79] Pauwels, E.J.; Salah, A.A.; Tavenard, R.; , "Sensor Networks for Ambient Intelligence," Multimedia Signal Processing, 2007. MMSP 2007. IEEE 9th Workshop on , vol., no., pp.13-16, 1-3 Oct. 2007

[80] Jaspal S. Sandhu. Wireless sensor networks for commercial lighting control: Decision making with multi-agent systems. In In AAAI Workshop on Sensor Networks, volume 10, pages 131-140, 2004.

[81] Jin He, Huaming Li, and Jindong Tan. Real-time daily activity classification with wireless sensor networks using hidden markov model. In 2007 29th Annual International Conference of the IEEE Engineering in Medicine and Biology Society, pages 3192-3195. IEEE, August 2007.

[82] Panu Korpipaa ; and Jani M, “ An ontology for mobile device sensor-based context awareness" In Proceedings of the 4th international and interdisciplinary conference on Modeling and using context (CONTEXT'03)

[83] Laurent Gomez, Annett Laube, and Alessandro Sorniotti. 2008. Design guidelines for integration of wireless sensor networks with enterprise systems. In Proceedings of the 1st international conference on MOBILe Wireless MiddleWARE, Operating Systems, and Applications (MOBILWARE '08). Article 12, 7 pages.

[84] P. Neves, J. Rodrigues, "Internet Protocol over Wireless Sensor Networks ,from Myth to Reality”, In Journal of Communications, Vol. 5, No. 3, March 2010.

[85] A. Liutkevicius, A. Vrubliauskas and E. Kazanavicius, "A Survey of Wireless Sensor Network Interconnection to External Networks" Novel Algorithms and Techniques in Telecommunications and Networking, (2010) pp 4146.

[86] Mittal Sangeeta and Maskara S.L., "Towards development of Wireless Sensor Network Services Based on Semantic Web", in proceedings of INDICON, 2010,Kolkata, India.

[87] Ghataoura, D.S.; Mitchell, J.E.; Matich, G.E.; , "Networking and application interface technology for wireless sensor network surveillance and monitoring," Communications Magazine, IEEE , vol.49, no.10, pp.90-97, Oct. 2011.

[88] Smith, J.M.; , "The doctor will see you ALWAYS," Spectrum, IEEE , vol.48, no.10, pp.56-62, October 2011 doi: 10.1109/MSPEC.2011.6027250 
Ms. Sangeeta Mittal is Senior Lecturer in Computer Science at the Department of Computer Science \& Information Technology, Jaypee Institute University, Noida, India. In teaching, she has been focusing on cryptography and network security. In research, her current interests include Wireless Sensor Networks, Context Aware Systems and Sensor based Smart Environments. Ms. Mittal received her Master degree in Computer Engineering from Punjab University Chandigarh India and BE from Maharishi Dayanand University, Rohtak, India. She is a member of IEEE and ACM.

Dr. Alok Aggarwal is Assistant Professor in Computer Science at the Department of Computer Science \& Information Technology, Jaypee Institute University, Noida, India. His current research interests are in areas of Mobile Computing and Object Oriented Programming. He has been working as Software Developer, Researcher and Teacher for past 14 years. He has published three books and about 40 Research papers in referred journals and Conf. Proceedings. He completed his Bachelors, Masters and $\mathrm{PhD}$ (Mobile Computing) in Computer Science and Engineering in 1995, 2001 and 2010 respectively.

Prof. S.L. Maskara is Life Senior Member of the IEEE, Life Fellow of the IETE, Life Fellow of the IE (India), Life Senior Member of the CSI, Life Member of the ISTE. He obtained his Ph.D. in Electronics \& Electrical Comm. Engg from IIT Kharagpur and M.E. in Electrical Comm Engg from IISc Bangalore. His research interests include Systems \& Networks; Wireless, Mobile, Satellite, Optical \& Computer Communications \& Networking; Engineering Education including Curriculum Planning and Design. He has a long teaching \& research experience of 46 years. He worked as Dean Ac \& Res at JIIT Noida 2006-11; Dist Prof. at LNMIIT Jaipur \& DAIICT Gandhinagar and Lect./Asst Prof./Prof. in the E\&ECE at IIT Kharagpur. 